Supplement of Hydrol. Earth Syst. Sci., 20, 4457-4467, 2016

http://www.hydrol-earth-syst-sci.net/20/4457/2016/

doi:10.5194/hess-20-4457-2016-supplement

(C) Author(s) 2016. CC Attribution 3.0 License.

(c) (i)

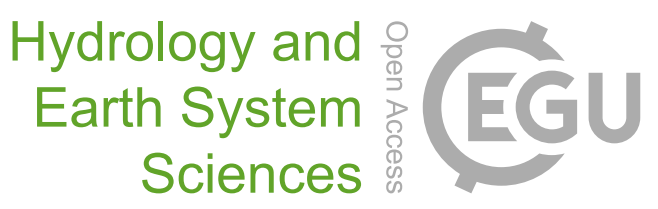

Supplement of

\title{
Hydrologic controls on aperiodic spatial organization of the ridge-slough patterned landscape
}

Stephen T. Casey et al.

Correspondence to: Matthew J. Cohen (mjc@ufl.edu)

The copyright of individual parts of the supplement might differ from the CC-BY 3.0 licence. 


\section{Supplementary maps}

To corroborate the results from the primary map (M1), we used two additional maps, M2 and M3 (Fig. S1). The spatial extent of M2 is similar to M1 and same site locations were used. Although rotation for flow-alignment can induce significant resampling error with larger cells, we found strong agreement between spatial statistics from M2 and an un-rotated version; thus, we used the same rotation scheme for M2 as for M1. The source map for M3 was generated from $1 \mathrm{~m}$ resolution digital orthophotos and each site was individually rotated in the direction of elongation (Nungesser, 2011). The spatial extent of the source map was composed of a series of non-adjacent $4 \mathrm{~km} \times 6 \mathrm{~km}$ blocks, and correspondingly we chose $104 \mathrm{~km} \times 6 \mathrm{~km}$ blocks and rasterized at $1 \mathrm{~m}$ resolution.

The results from supplementary maps were consistent with the findings from the primary map. Ridge density relationships show strong agreement (Fig. S2), with similar east west trends observed in all maps. Likewise, elongation shows congruent relationships across maps (Fig. S3). Note that the anisotropic extent of M3 sites may bias those measurements slightly in favor of higher elongation values compared to sites with square extents (i.e. M1 and M2).

Edge density was positively correlated to MWD for both high resolution maps (M1, M3), indicating greater perimeter at deeper sites (Fig. S4a \& S4c). In contrast, M2 shows a relationship consistent with a significant loss of edge detail due the map's low sampling resolution of 50m (Fig S4b). Figure S4e shows that edge density is consistent with a null model, where edge density is maximized when occupied cells reach a density of 0.5 , and smoothly declines to zero as density approaches zero or one (Gardner et al., 1987). This pattern is clear for M2, suggesting ridge density alone controls perimeter values, not an underlying hydrologic phenomenon. In contrast, the variation observed in M1 and M3 appear much less consistent with 
the null model (Fig S4d) relative to the MWD relationship. Notably, when M1 was resampled to $50 \mathrm{~m}$ cells, perimeter was strongly controlled by ridge density. This discrepancy between lowand high-resolution maps suggests that the MWD vs. perimeter relationship is manifest principally as variation in fine-scale geometry.

\section{Patch scaling relationships}

In addition to patch size distributions, we also looked at how shape attributes change across patch size. Patch scaling relationships were identified by regressing patch size against patch-level perimeter and elongation. Patch perimeter was the sum of edge cells for a given patch. Patch elongation (Eq. 1), is applied to cells belonging to an individual patch, rather than the landscape as a whole (as for site-site comparisons). We omit sites with less than 100 observations.

We found that although patch area distributions indicate scale invariance, patch scaling relationships show some scale dependence with patch size. The association between patch perimeter and area was better approximated (F-statistic <0.001) by quadratic rather than linear perimeter-to-area scaling on log-log plots (Fig. S5a), indicating larger patches have higher perimeters than expected from a fractal relationship. Likewise, a significant positive association $(\mathrm{p}<0.05)$ between patch elongation and patch size was observed in most sites ( $p$-values in Table S1), indicating that patches become more elongated with size (Fig. S5b). The significance of these results is somewhat unclear however, as the behavior of patch-based elongation for complex, non-Euclidean shapes is relatively unexplored. Correspondingly, we emphasize some caution in the interpretation of these results, particularly in light of the relatively weak correlations observed. Nonetheless, we find that the consistency of this result (i.e. patch-based 
elongation having a positive correlation with patch size) across a wide range of sites compelling, which may point to an intrinsic property of the landscape features.

The finding that patch elongation increases with patch size indicates that patches are not strictly self-similar. Although the patch elongation scaling may result from scale-dependent mechanisms (i.e., processes that impose a characteristic spatial scale) it may also suggest anisotropic scale-free processes that produce self-affinity, a fractal form where geometric features scale via separate relations in the $\mathrm{x}$ and $\mathrm{y}$ directions. Self-affinity implies that lengths and widths are scale invariant with respect to their orthogonal directions, however the patches as a whole only become scale invariant when an anisotropic rescaling transformation is made (Sapozhnikov and Foufoula-Georgiou, 1996).Patch perimeter also shows departure from fractal scaling, with patches increasing in perimeter more rapidly with size than expected from a simple fractal relationship. The mechanisms driving this are unclear. Multiple processes can induce large patches to form greater than expected perimeters, such as the increased elongation, weblike connectivity, or increased crenulation. Conversely, this curvature may be due to artifacts such as the mapping scheme capturing a greater proportion of the relative detail for larger patches relative to small patches. Resolution limitations become more apparent on small-scale features when mapped at the same resolution as large scale features. Detail-sensitive measurements like perimeter may be skewed according to relative patch scale such that, at lowresolution, small patches are represented as more Euclidean shapes (i.e. having boxier edges), while larger patches retain more complex representations, creating artificial scale dependence. However, while inference of intrinsic perimeter scaling relations may be problematic because of these map resolution effects, this effect is unlikely to explain variation in patch elongation with patch size because small patches are free to achieve very high elongation values (e.g. the cutoff 
patch size for M1 of $100 \mathrm{~m}$ is capable of producing elongation values up to 100, well above any observed value).

\section{Patch size distributions}

For the Monte Carlo simulations we used a reweighted form of the Kolmogorov-Smirnov (KS) test (Anderson and Darling, 1952):

$$
\max _{\mathrm{x} \geq \mathrm{x}_{\min }} \frac{|\mathrm{S}(\mathrm{x})-\mathrm{P}(\mathrm{x})|}{\sqrt{\mathrm{P}(\mathrm{x})(1-\mathrm{P}(\mathrm{x}))}}
$$

where $\mathrm{S}(\mathrm{x})$ is the empirical CCDF and $\mathrm{P}(\mathrm{x})$ is the continuous CCDF of the candidate distribution, fit to the data using maximum likelihood estimation (MLE). In comparing the GP to the lognormal, we were particularly interested in the behavior at the tail end of the distribution, as the lognormal CCDF deviates from a power-law for very large x (Malevergne et al., 2011). The standard KS test is relatively insensitive to tail differences as the CCDF approaches zero; the reweighting scheme remedies this issue.

We compared the candidate distribution fits by generating log-likelihood ratios of modeled distributions and calculating significance values (Clauset et al., 2009). We reject the hypothesis that the empirical data is consistent with a candidate distribution at $\mathrm{p}<0.1$ for the Monte Carlo tests and at $\mathrm{p}>0.1$ for the log-likelihood ratios. The minimum patch size used to create the empirical distributions was chosen to balance between omitting potentially biased values (which may cause us to reject an otherwise valid distribution) and providing a sample size sufficient for accurate parameter estimation. For $\mathrm{M} 1, \mathrm{x}_{\min }=100 \mathrm{~m}^{2}$ was chosen. However, this cutoff appeared too low for M3, as that map had significantly fewer patches than predicted by both the GP and lognormal in the $100-500 \mathrm{~m}^{2}$ range; correspondingly we chose $\mathrm{x}_{\mathrm{min}}=500 \mathrm{~m}^{2}$ for M3. For both M1 and M3, we omit any sites with fewer than 100 observations. 
We note that while the GP is a continuous distribution, raster maps yield discrete data corresponding to the cell size. Where the cells are much smaller than $\mathrm{x}_{\text {min }}$, discretization effects are negligible. However, as the cell size approaches $\mathrm{x}_{\min }$, discretization can create significant bias. We approximated a discrete version of the GP using the continuous form, assuming that each discrete value represents a continuous data point from $(x-$ cellsize/ 2$)$ to $(x+$ cellsize/2). The

corrected $x_{\min }$ value, $x_{\min }^{*}$, becomes $\left(x_{\min }-\right.$ cellsize/2) (e.g., for $M 1$, and $\left.x_{\min }^{*}=99.5 \mathrm{~m}^{2}\right)$. Synthetic data sets were rounded to the nearest discretization level to be consistent. A similar correction for the power-law distribution worked well when $\mathrm{x}_{\min }$ was 6-fold larger than the discretization level (i.e., cell size) (Clauset et al., 2009). For maps with a $1 \mathrm{~m}$ cell size, $\mathrm{x}_{\min }$ was two orders of magnitude greater than the cell size, and we found no significant difference between corrected and uncorrected results. However, for maps with larger cell sizes, discretization effects caused significant error, suggesting the continuous form approximation may not be appropriate. As such, we omitted M2 from patch size distribution analysis.

\section{Distances between ridge centers}

In addition to spectral analysis and patch size distributions, we also investigated distances between ridge centers as evidence of periodicity. Ridge centers were defined as the center point of every contiguous group of ridge cells along each lateral row or longitudinal column. Distances from each center to their neighboring centers was calculated, and the corresponding distributions for both lateral and longitudinal directions was generated (Fig. S6).

All sites show a distinct mode (between 25 and $75 \mathrm{~m}$ for the high pixel resolution blocks), with nearly identical longitudinal and lateral mode distances (Fig. S6a, S6b). The modes for high resolution sites (e.g., 5, 11, 14, 20) is $\sim 65 \mathrm{~m}$, while sites mapped at lower resolution 
(e.g., 2, 25) had modes $\sim 160 \mathrm{~m}$. Highly degraded sites had slightly lower modes ( 25-50m). Differences between low and high resolution sites suggest that mode values are highly dependent on mapping resolution; indeed, when we experimentally lower map resolution, we see a consistent modal shifting to higher values (Fig. S6c), suggesting that smaller features (i.e., $<75 \mathrm{~m}$ for high resolution sites and $<150 \mathrm{~m}$ for low resolution sites) are underrepresented at lower resolution. We note that the observed mode values are consistent with the map's stated resolution limitations (Rutchey 2005). Lower modes in degraded sites is consistent with the clear patch disaggregation process that we visually observe, where large ridges are fragmented. However, without maps that exceed the currently available resolution, assessing whether observed modes are actual landscape features or mapping artefacts is challenging. Indeed, undersampling of small features (i.e. short distances between ridges) due to limited resolution will artificially produce a mode near the resolution limit, regardless of whether one actually exists in the landscape. Therefore, the observation of modes within this region should not be used to verify or refute the presence of periodicity. 


\section{References}

Anderson, T. W. and Darling, D. A.: Asymptotic theory of certain "goodness of fit" criteria based on stochastic processes, Ann. Math. Stat., 23, 193-212, 1952.

Clauset, A., Shalizi, C. R., and Newman, M. E.: Power-law distributions in empirical data, SIAM Rev., 51, 661-703, 2009.

Jones, D. M., Madden, M., Snyder, J., and Rutchey, K.: A Vegetation Classification System for Southern Florida's National Parks and Preserves, South Florida Natural Resources Center Technical Report, South Florida Natural Resources Center, Everglades National Park, National Park Service, U.S. Department of the Interior, Homestead, Florida. 1999.

Gardner, R. H., Milne, B. T., Turner, M. G., and O’Neill, R. V.: Neutral models for the analysis of broad-scale landscape pattern, Landscape Ecol., 1, 19-28, 1987.

Malevergne, Y., Pisarenko, V., and Sornette, D.: Testing the Pareto against the lognormal distributions with the uniformly most powerful unbiased test applied to the distribution of cities, Phys. Rev. E, 83, 036111, 2011.

Nungesser, M. K.: Reading the landscape: temporal and spatial changes in a patterned peatland, Wetl. Ecol. Manag., 19, 475-493, 2011.

Rutchey, K., Vilchek, L., and Love, M.: Development of a vegetation map for Water Conservation Area 3, Technical Publication ERA Number 421, South Florida Water Management District, West Palm Beach, FL, USA, 2005.

Sapozhnikov, V. and Foufoula-Georgiou, E.: Self-affinity in braided rivers, Water Resour. Res., 32, 1429-1439, 1996. 


\section{Table Legends}

Table S1. Selected site properties for all maps (M1, M2, M3). Mean patch angle ( $\left.A_{p}\right)$ measures the angular difference between patch and site orientation. The $\mathrm{p}$ value for patch $\mathrm{L}: \mathrm{W}$ indicates the significance of a linear regression relating patch length-to-width to area for M1. Spearman correlation, $\rho$, indicates the monotonicity of observed $r$ spectrum plots with a value of -1 corresponding to a perfectly monotonic function; $\rho$ values for the full and directional $r$ spectra were $<-0.999$ and $<-0.99$ (not shown), respectively, for all sites in M1 and M3 confirming the absence of a characteristic pattern wavelength. Both directional and omnidirectional r-spectra were well approximated by a power law, with all sites in M1 having $\mathrm{R}^{2}$ greater than .99 and .98 respectively, and all sites in M3 having $\mathrm{R}^{2}$ greater than .99 for both types of r-spectra (not shown). $\rho$ values and $\mathrm{R}^{2}$ values for M2 were less significant due to the reduced sample size. NA values correspond to sites that were omitted from patch-level analyses because they contained less than 100 patches.

Table S2. Generalized Pareto and truncated log-normal tests for patch area distributions for M1 and M3. For each site, we give the total number of data points in each empirical distribution, $\mathrm{n}$, along with the maximum patch area, $\max \mathrm{x}$. For the Monte Carlo p-values panel, we give the corresponding p-values for the Monte Carlo tests. Significant values ( $p$ $>0.1$ ) are denoted in bold, and represent a data set being consistent with Monte Carlo tests (site $\mathrm{j}$ for the log-normal did not converge on a result). $\mathrm{p}$-values corresponding to the log-likelihood ratio tests are also given, with statistically significant values $(\mathrm{p}<0.1)$ represented in bold (if a value is significant, it is considered a poor fit in comparison to the alternate distribution). The panel on the right gives parameter values corresponding to the GP.

Table S3. Classification scheme for binary maps. For M1 and M2, vegetation classes in the original maps were converted to a binary representation (note that the source map for M3 was already represented as binary values and required no conversion). Value represents the corresponding binary class (Slough $=0$; Ridge $=1$ ). Area gives the total areal coverage of each vegetation ID for the original map extent. ID gives the vegetation ID corresponding to the original map's classification system. For full descriptions of each vegetation ID, see Jones et al (1999) for M1 and Rutchey et al (2005) for M2. 


\section{Figure Legends}

Figure S1. Supplementary maps and site locations. Note that M2 shares the same site locations as M1.

Figure S2. Ridge density relationships were consistent across all maps. Eastern and western partitions show similar behavior across maps as well.

Figure S3. Site elongation relationships. Sites with ridge densities greater than 0.8 (indicated in grey) were omitted. Site 1 was omitted due to possible misclassification issues.

Figure S4. Relationship between edge density and both MWD and ridge density for all maps. (a, c) Edge density is positively correlated to mean water depth. Higher edge densities in deeper sites is consistent with patches becoming disaggregated with increased water depth. (b) M2 shows a significantly weaker relationship, which can be explained by a loss of edge detail due to the low resolution of M2. (e) This is illustrated by a peak in edge density when ridge and slough heterogeneity is maximized (i.e., ridge density $=0.5$ ) and a minimum when heterogeneity is minimized (i.e., ridge density $=0$ or 1 ) suggesting edge density is controlled by ridge density rather than the underlying hydrology. M2 is consistent with this null hypothesis, while M1 and M3 both have significant hydrologic trends. When M1 was resampled to $50 \mathrm{~m}$ pixels (not shown), the relationship was similar to $\mathrm{M} 2$, indicating that larger pixel sizes do not to capture the scale of features responsible for this correlation. Gray sites in M1 indicate those mapped at lower resolution, and omitted from regressions.

Figure S5. (a) Patch elongation (log-transformed length to width ratio) increases with patch size, with a weaker relationship in highly inundated sites $(14,28)$. (b) Perimeter to area scaling shows a slight curvature (linear model is shown in blue; quadratic model in red) indicating that patch perimeter increases faster than expected for a fractal relationship.

Figure S6: (a) Histograms of distances between ridge centers in the lateral direction were approximated by a Gamma distribution (indicated in blue) and show a distinct mode ( $\varphi$; indicated in red). The location of this mode is at an average of $64 \mathrm{~m}$ for high resolution sites (e.g., 5, 11, 20, 14) and $162 \mathrm{~m}$ for low resolution sites (e.g., 2, 25). In minimally impacted (sites 3-8;10-12;15,16), $\varphi$ averaged 78 m. (b) Histogram of distances between ridge centers in the longitudinal direction show similar forms and mode locations compared to lateral distances, with mean $\varphi$ of $57 \mathrm{~m}$ in high resolution sites and $162 \mathrm{~m}$ in 
low resolution sites. Minimally impacted sites had a mean $\varphi$ of $69 \mathrm{~m}$. (c) To illustrate the effects of mapping resolution on the emergence of modes, we resampled site 11 to sequentially lower resolution (effective pixel size, $\varepsilon$, of $10 \mathrm{~m}, 20 \mathrm{~m}, 30 \mathrm{~m}, 40 \mathrm{~m}$, and 50 $\mathrm{m})$ and generated corresponding lateral histograms. The mode increases with decreased resolution, as fine scale features are lost. This suggests that the modes present in both lateral and longitudinal analyses may be artefacts of the mapping process, rather than real features in the landscape, a point underscored by observing that computed modes are always below the stated mapping resolution.

Figure S7: (a) Lateral $r$ spectra for all sites, arranged along a gradient from wet (upper left) to dry (bottom right). The form of the $r$ spectra shows a mixture of exponential and power law behaviors, with higher wavenumbers exhibiting generally power-law (linear in loglog space) behavior, with rounding towards an exponential function at lower wavenumbers. Sites which visually show clearly defined patterning (e.g. sites 2-8; 10-13; $15,16,18,20)$ exhibit clear demarcation between the linear and curved portions of the $r$ spectra, while the demarcation is spread across a greater range of wave numbers in degraded sites. (b) Longitudinal $r$ spectra for all sites are arranged along the hydrologic gradient. Similar to lateral $r$ spectra, the form is a mixture of power law and exponential, although in general, the curvature for the longitudinal spectra appears at a wider range of wavenumbers.

Table S1 


\begin{tabular}{|c|c|c|c|c|c|c|c|c|c|c|c|c|}
\hline \multicolumn{3}{|c|}{ M1 } & \multicolumn{7}{|c|}{ M2 } & \multicolumn{3}{|c|}{ M3 } \\
\hline$\stackrel{ \pm}{*}$ & $\begin{array}{l}\frac{0}{80} \\
\frac{0}{0} \\
\frac{0}{4}\end{array}$ & 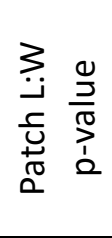 & $\stackrel{⿱}{*}$ & $\begin{array}{l}\frac{0}{00} \\
\frac{0}{0} \\
\frac{0}{4}\end{array}$ & 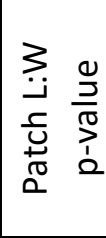 & 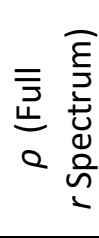 & 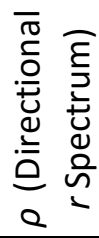 & 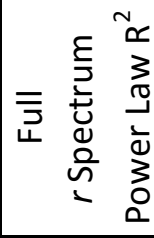 & 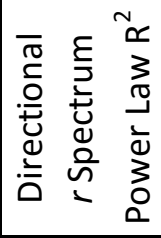 & $\stackrel{⿱}{*}$ & $\begin{array}{l}\frac{0}{00} \\
\frac{0}{0} \\
\frac{0}{4}\end{array}$ & 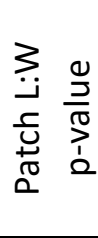 \\
\hline 1 & -5.7 & 0.13 & 1 & -0.5 & $<0.01$ & -0.97 & -0.95 & 0.97 & 0.96 & $a$ & 2.8 & $<0.01$ \\
\hline 2 & 1.8 & 0.05 & 2 & -0.4 & $<0.01$ & -0.98 & -0.95 & 0.97 & 0.92 & b & N/A & N/A \\
\hline 3 & 2.7 & $<0.01$ & 3 & 4.5 & $<0.01$ & -0.98 & -0.97 & 0.99 & 0.97 & c & 1.2 & $<0.01$ \\
\hline 4 & -1.2 & $<0.01$ & 4 & 0.2 & $<0.01$ & -0.97 & -0.86 & 0.99 & 0.78 & $d$ & -7.2 & $<0.01$ \\
\hline 5 & -3.9 & $<0.01$ & 5 & 3.6 & $<0.01$ & -0.98 & -0.94 & 0.96 & 0.89 & e & 0.7 & $<0.01$ \\
\hline 6 & 0.9 & $<0.01$ & 6 & 2.3 & $<0.01$ & -0.98 & -0.95 & 0.97 & 0.87 & $f$ & -0.7 & $<0.01$ \\
\hline 7 & -2.4 & $<0.01$ & 7 & 0.6 & $<0.01$ & -0.99 & -0.96 & 0.99 & 0.90 & g & -5.0 & 0.01 \\
\hline 8 & -3.6 & $<0.01$ & 8 & 2.0 & $<0.01$ & -0.98 & -0.94 & 0.99 & 0.88 & $\mathrm{~h}$ & -1.4 & $<0.01$ \\
\hline 9 & -2.2 & 0.32 & 9 & 8.5 & $<0.01$ & -0.96 & -0.96 & 0.97 & 0.95 & i & 3.2 & $<0.01$ \\
\hline 10 & -1.5 & $<0.01$ & 10 & 2.6 & $<0.01$ & -0.96 & -0.93 & 0.91 & 0.80 & j & -3.3 & 0.03 \\
\hline 11 & 0.4 & $<0.01$ & 11 & 2.7 & $<0.01$ & -0.97 & -0.94 & 0.97 & 0.85 & & & \\
\hline 12 & -3.8 & $<0.01$ & 12 & 0.5 & $<0.01$ & -0.98 & -0.89 & 0.98 & 0.79 & & & \\
\hline 13 & 0.5 & 0.21 & 13 & 2.0 & $<0.01$ & -0.97 & -0.96 & 0.99 & 0.90 & & & \\
\hline 14 & -0.3 & $<0.01$ & 14 & 4.1 & $<0.01$ & -0.96 & -0.92 & 0.99 & 0.92 & & & \\
\hline 15 & -4.5 & $<0.01$ & 15 & 2.6 & $<0.01$ & -0.98 & -0.96 & 0.95 & 0.91 & & & \\
\hline 16 & -3.1 & $<0.01$ & 16 & 0.9 & $<0.01$ & -0.97 & -0.96 & 0.99 & 0.92 & & & \\
\hline 17 & -0.1 & $<0.01$ & 17 & 1.2 & $<0.01$ & -0.97 & -0.95 & 0.99 & 0.94 & & & \\
\hline 18 & -2.7 & $<0.01$ & 18 & 5.2 & $<0.01$ & -0.98 & -0.93 & 0.99 & 0.94 & & & \\
\hline 19 & -4.0 & $<0.01$ & 19 & 0.8 & $<0.01$ & -0.96 & -0.85 & 0.98 & 0.74 & & & \\
\hline 20 & -4.0 & $<0.01$ & 20 & -1.5 & $<0.01$ & -0.99 & -0.87 & 0.98 & 0.85 & & & \\
\hline 21 & 2.5 & $<0.01$ & 21 & 1.0 & $<0.01$ & -0.98 & -0.92 & 0.98 & 0.87 & & & \\
\hline 22 & -6.4 & $<0.01$ & 22 & 0.3 & $<0.01$ & -0.98 & -0.90 & 0.98 & 0.93 & & & \\
\hline 23 & N/A & N/A & 23 & $\mathrm{~N} / \mathrm{A}$ & N/A & -0.98 & -0.91 & 0.98 & 0.93 & & & \\
\hline 24 & N/A & N/A & 24 & N/A & N/A & -0.94 & -0.91 & 0.97 & 0.92 & & & \\
\hline 25 & N/A & N/A & 25 & 14.7 & 0.35 & -0.97 & -0.89 & 0.98 & 0.85 & & & \\
\hline 26 & N/A & N/A & 26 & $\mathrm{~N} / \mathrm{A}$ & N/A & -0.95 & -0.90 & 0.97 & 0.94 & & & \\
\hline 27 & N/A & N/A & 27 & 5.3 & 0.57 & -0.98 & -0.94 & 0.98 & 0.90 & & & \\
\hline 28 & -2.6 & 0.33 & 28 & 2.7 & $<0.01$ & -0.98 & -0.95 & 0.99 & 0.97 & & & \\
\hline 29 & -2.3 & 0.10 & 29 & 5.0 & $<0.01$ & -0.96 & -0.90 & 0.98 & 0.95 & & & \\
\hline 30 & N/A & $\mathrm{N} / \mathrm{A}$ & 30 & N/A & N/A & -0.95 & -0.86 & 0.98 & 0.88 & & & \\
\hline 31 & 3.1 & 0.09 & 31 & 5.1 & 0.31 & -0.97 & -0.93 & 0.98 & 0.95 & & & \\
\hline 32 & N/A & N/A & 32 & $\mathrm{~N} / \mathrm{A}$ & N/A & -0.97 & -0.87 & 0.98 & 0.85 & & & \\
\hline 33 & N/A & $\mathrm{N} / \mathrm{A}$ & 33 & -3.3 & 0.49 & -0.96 & -0.75 & 0.96 & 0.46 & & & \\
\hline
\end{tabular}

Table S2 


\begin{tabular}{|c|c|c|c|c|c|c|c|c|c|c|c|c|c|}
\hline \multirow[b]{2}{*}{$\stackrel{ \pm}{5}$} & & \multirow[b]{2}{*}{ 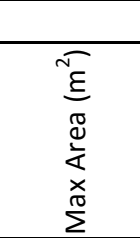 } & \multirow[b]{2}{*}{ 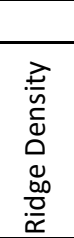 } & \multicolumn{2}{|c|}{ Monte Carlo $p$} & \multicolumn{2}{|c|}{ Likelihood $p$} & \multicolumn{6}{|c|}{ GP Parameters } \\
\hline & & & & i & 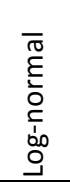 & i & $\begin{array}{l}\overline{\widetilde{T}} \\
\underline{\xi} \\
\overline{0} \\
\stackrel{0}{0.0} \\
\stackrel{.}{0}\end{array}$ & $\delta$ & $95 \% \mathrm{Cl}(\delta)$ & $k$ & $95 \% \mathrm{Cl}(k)$ & $\alpha^{*}$ & $95 \% \mathrm{Cl}\left(\alpha^{*}\right.$ \\
\hline 1 & 428 & 7216732 & 0.51 & 0.04 & 0.00 & 1.00 & 0.43 & 4423 & $3732-5242$ & 0.84 & $0.68-1.00$ & 2.19 & $2.00-2.48$ \\
\hline 2 & 225 & 16080806 & 0.64 & 0.02 & 0.00 & 1.00 & 0.17 & 2999 & $2379-3781$ & 0.96 & $0.73-1.19$ & 2.04 & $1.84-2.37$ \\
\hline 3 & 1414 & 9973159 & 0.58 & 0.80 & 0.00 & 1.00 & 0.10 & 719 & 643-803 & 1.24 & $1.13-1.36$ & 1.80 & $1.73-1.89$ \\
\hline 4 & 1214 & 5192468 & 0.61 & 0.62 & 0.01 & 1.00 & 0.00 & 534 & $474-602$ & 1.30 & $1.17-1.42$ & 1.77 & $1.70-1.86$ \\
\hline 5 & 757 & 23399024 & 0.73 & 0.02 & 0.00 & 1.00 & 0.08 & 523 & $454-603$ & 1.02 & $0.88-1.16$ & 1.98 & $1.86-2.14$ \\
\hline 6 & 1476 & 3137995 & 0.54 & 0.13 & 0.04 & 1.00 & 0.01 & 558 & $500-624$ & 1.33 & $1.21-1.45$ & 1.75 & $1.69-1.82$ \\
\hline 7 & 1083 & 4205933 & 0.61 & 0.05 & 0.00 & 1.00 & 0.00 & 711 & $631-802$ & 1.17 & $1.05-1.30$ & 1.85 & $1.77-1.95$ \\
\hline 8 & 1063 & 18466609 & 0.65 & 0.08 & 0.00 & 1.00 & 0.23 & 402 & $354-457$ & 1.13 & $1.00-1.26$ & 1.89 & $1.79-2.00$ \\
\hline 9 & 685 & 25113931 & 0.72 & 0.00 & 0.00 & 1.00 & 0.41 & 414 & $358-477$ & 0.76 & $0.62-0.89$ & 2.32 & $2.12-2.60$ \\
\hline 10 & 1414 & 2173573 & 0.52 & 0.55 & 0.03 & 1.00 & 0.41 & 642 & 571-722 & 1.37 & $1.24-1.50$ & 1.73 & $1.67-1.80$ \\
\hline 11 & 1418 & 12266427 & 0.56 & 0.10 & 0.00 & 1.00 & 0.01 & 534 & $482-592$ & 1.03 & $0.92-1.13$ & 1.98 & $1.89-2.09$ \\
\hline 12 & 1099 & 19428337 & 0.64 & 0.12 & 0.00 & 1.00 & 0.16 & 357 & $316-403$ & 1.17 & $1.05-1.30$ & 1.85 & $1.77-1.96$ \\
\hline 13 & 1187 & 19053356 & 0.64 & 0.05 & 0.00 & 1.00 & 0.17 & 329 & 293-371 & 1.09 & $0.97-1.21$ & 1.92 & $1.83-2.03$ \\
\hline 14 & 1877 & 8407083 & 0.49 & 0.20 & 0.00 & 1.00 & 0.12 & 456 & 415-502 & 1.18 & $1.08-1.28$ & 1.85 & $1.78-1.92$ \\
\hline 15 & 1693 & 2307671 & 0.46 & 0.53 & 0.13 & 0.96 & 1.00 & 432 & 388-481 & 1.43 & $1.31-1.54$ & 1.70 & $1.65-1.76$ \\
\hline 16 & 2092 & 2387455 & 0.48 & 0.46 & 0.13 & 0.38 & 1.00 & 288 & 261-317 & 1.44 & $1.34-1.55$ & 1.69 & $1.65-1.75$ \\
\hline 17 & 2453 & 3797668 & 0.48 & 0.77 & 0.02 & 1.00 & 0.45 & 302 & $277-3$ & 1.27 & $1.18-1$ & 1.79 & $1.73-1.85$ \\
\hline 18 & 2367 & 3956762 & 0.47 & 0.40 & 0.10 & 1.00 & 0.24 & 302 & 30 & 1.39 & $1.30-1$ & 1.72 & -1.77 \\
\hline 19 & 2132 & 3442071 & 0.46 & 0.33 & 0.28 & 0.63 & 1.00 & 265 & 2 & 1.46 & $1.35-$ & 1.69 & -1.74 \\
\hline 20 & 1765 & 2482129 & 0.47 & 0.20 & 0.05 & 1.00 & 0.07 & 405 & & 1.36 & 1.25 & 1.73 & 1.80 \\
\hline 21 & 1623 & 1876363 & 0.48 & 0.65 & 0.13 & 1.00 & 0.33 & 61 & & 1.40 & $1.28-$ & 1.71 & $1.66-1.78$ \\
\hline 22 & 2486 & 4207373 & 0.51 & 0.19 & 0.09 & 0.95 & 1.00 & 285 & $261-$ & 1.35 & $1.26-1.45$ & 1.74 & $1.69-1.79$ \\
\hline 23 & 35 & 31517817 & 0.88 & N/A & N/A & N/A & N/A & N/A & N/A & N/A & N/A & N/A & N/A \\
\hline 24 & 4 & 35833341 & 1.00 & N/A & N/A & N/A & N/A & $\mathrm{N} / \mathrm{A}$ & $\mathrm{N} / \mathrm{A}$ & N/A & N/A & N/A & $\mathrm{N} / \mathrm{A}$ \\
\hline 25 & 16 & 34210049 & 0.95 & N/A & N/A & $\mathrm{N} / \mathrm{A}$ & N/A & $\mathrm{N} / \mathrm{A}$ & $\mathrm{N} / \mathrm{A}$ & $N / A$ & N/A & N/A & $\mathrm{N} / \mathrm{A}$ \\
\hline 26 & 21 & 33117463 & 0.93 & $N / A$ & N/A & $\mathrm{N} / \mathrm{A}$ & N/A & $\mathrm{N} / \mathrm{A}$ & $\mathrm{N} / \mathrm{A}$ & N/A & $\mathrm{N} / \mathrm{A}$ & N/A & $\mathrm{N} / \mathrm{A}$ \\
\hline 27 & 42 & 21551629 & 0.62 & N/A & N/A & N/A & N/A & N/A & $\mathrm{N} / \mathrm{A}$ & $\mathrm{N} / \mathrm{A}$ & $\mathrm{N} / \mathrm{A}$ & N/A & $\mathrm{N} / \mathrm{A}$ \\
\hline 28 & 640 & 22853504 & 0.69 & 0.03 & 0.00 & 1.00 & 0.36 & 494 & $421-580$ & 1.09 & $0.93-1.26$ & 1.91 & $1.80-2.08$ \\
\hline 29 & 898 & 7165571 & 0.48 & 0.41 & 0.01 & 1.00 & 0.09 & 887 & 771-1022 & 1.31 & $1.16-1.46$ & 1.76 & $1.68-1.86$ \\
\hline 30 & 97 & 32129324 & 0.90 & N/A & $\mathrm{N} / \mathrm{A}$ & N/A & N/A & N/A & N/A & N/A & N/A & N/A & N/A \\
\hline 31 & 331 & 29628741 & 0.83 & 0.00 & 0.00 & 1.00 & 0.12 & 415 & $345-500$ & 0.80 & $0.62-0.97$ & 2.25 & $2.03-2.60$ \\
\hline 32 & 69 & 33363362 & 0.93 & N/A & $\mathrm{N} / \mathrm{A}$ & N/A & N/A & N/A & N/A & N/A & $\mathrm{N} / \mathrm{A}$ & N/A & N/A \\
\hline 33 & 51 & 32409533 & 0.90 & N/A & $\mathrm{N} / \mathrm{A}$ & $\mathrm{N} / \mathrm{A}$ & N/A & N/A & N/A & N/A & N/A & N/A & $\mathrm{N} / \mathrm{A}$ \\
\hline a & 216 & 15299260 & 0.73 & 0.03 & 0.00 & 1.00 & 0.09 & 1497 & $1150-1948$ & 1.07 & $0.80-1.34$ & 1.94 & $1.75-2.25$ \\
\hline b & 2 & 21829469 & 0.93 & N/A & $\mathrm{N} / \mathrm{A}$ & N/A & N/A & N/A & N/A & $\mathrm{N} / \mathrm{A}$ & N/A & $\mathrm{N} / \mathrm{A}$ & N/A \\
\hline c & 637 & 4011815 & 0.55 & 0.11 & 0.15 & 0.35 & 1.00 & 1770 & $1490-2103$ & 1.21 & $1.03-1.39$ & 1.83 & $1.72-1.97$ \\
\hline d & 230 & 15331726 & 0.73 & 0.01 & 0.00 & 1.00 & 0.00 & 1137 & 896-1444 & 0.93 & $0.69-1.16$ & 2.08 & $1.86-2.44$ \\
\hline e & 578 & 2948251 & 0.59 & 0.30 & 0.04 & 1.00 & 0.00 & 1566 & 1329-1846 & 1.11 & $0.95-1.28$ & 1.90 & $1.78-2.06$ \\
\hline$f$ & 423 & 4267675 & 0.65 & 0.03 & 0.01 & 1.00 & 0.00 & 1208 & $997-1$ & 1.14 & .94-1.34 & 1.87 & $1.74-2.06$ \\
\hline g & 795 & 1011669 & 0.39 & & 0.02 & 1.00 & 0.16 & 1275 & 1108-1469 & 1.02 & $0.87-1.16$ & 1.98 & $1.86-2.14$ \\
\hline $\mathrm{h}$ & 692 & 848690 & 0.41 & 0.38 & 0.47 & 0.15 & 1.00 & 1348 & $1145-1587$ & 1.32 & $1.15-1.50$ & 1.76 & $1.67-1.87$ \\
\hline $\mathrm{i}$ & 744 & 1323207 & 0.41 & 0.07 & 0.04 & 1.00 & 0.00 & 1926 & 1683-2204 & 0.97 & $0.83-1.10$ & 2.03 & $1.91-2.20$ \\
\hline & 142 & 19226070 & 0.83 & 0.00 & $\mathrm{~N} / \mathrm{A}$ & 1.00 & 0.39 & 631 & $472-844$ & 0.85 & $0.57-1.12$ & 2.18 & $1.89-2.75$ \\
\hline
\end{tabular}

Table S3 


\begin{tabular}{|c|c|c|c|c|c|}
\hline \multicolumn{3}{|c|}{ M1 } & \multicolumn{3}{|c|}{ M2 } \\
\hline Value & Count & VEG & Value & Count & VEG \\
\hline 0 & $6.44 \mathrm{E}+08$ & PGW & 0 & 262011 & MFO \\
\hline 0 & 37733353 & PEf & 0 & 5408 & MFGtM \\
\hline 0 & 635589 & PGe & 0 & 2587 & CStO \\
\hline 0 & 258091 & PGp & 0 & 314 & CSO \\
\hline 0 & 245020 & PGa & 0 & 43 & MFGh \\
\hline 0 & 217880 & PGx & 1 & 537835 & MFGc \\
\hline 0 & 171984 & PEo & 1 & 34929 & MFGtD \\
\hline 0 & 129254 & $w$ & 1 & 22954 & CSGc \\
\hline 1 & $1.41 E+09$ & PGc & 1 & 9117 & MFF \\
\hline 1 & $1.18 \mathrm{E}+08$ & PC & 1 & 5967 & MFG \\
\hline 1 & 6797153 & FSd & 1 & 3107 & MFGtS \\
\hline 1 & 4050063 & FSt & 1 & 2005 & CStD \\
\hline 1 & 4016163 & SBt & 1 & 1770 & CStGc \\
\hline 1 & 107552 & PEa & 1 & 527 & $\mathrm{MFH}$ \\
\hline 1 & 39287028 & SB & 1 & 448 & CStG \\
\hline 1 & 17165990 & SBs & 1 & 80 & WStG \\
\hline 1 & 16360398 & $\mathrm{SBm}$ & 1 & 13712 & SSs \\
\hline 1 & 8284266 & PE & 1 & 6909 & SSB \\
\hline 1 & 6573566 & $\mathrm{HI}$ & 1 & 5131 & SS \\
\hline 1 & 5126269 & FS & 1 & 4247 & FSt \\
\hline 1 & 4074153 & PEb & 1 & 2845 & CSE \\
\hline 1 & 1337204 & SBI & 1 & 2324 & MFB \\
\hline 1 & 771712 & EM & 1 & 1494 & FSB \\
\hline 1 & 442097 & SBa & 1 & 1039 & CSG \\
\hline 1 & 256461 & ES & 1 & 467 & SSm \\
\hline 1 & 9002 & SBC & 1 & 450 & SSI \\
\hline 1 & 2215 & $E$ & 1 & 164 & SSa \\
\hline 1 & 1582 & SBy & 1 & 140 & FHS \\
\hline 1 & 786 & EC & 1 & 132 & WStS \\
\hline 1 & 11057901 & SA & 1 & 61 & CStE \\
\hline 1 & 8989624 & C & 1 & 51 & EsD \\
\hline 1 & 2674872 & $\mathrm{RD}$ & 1 & 28 & SSy \\
\hline 1 & 81908 & SAd & 1 & 20 & FC \\
\hline 1 & 540 & $\mathrm{HIp}$ & 1 & 9 & DI \\
\hline & & & 1 & 6 & EsS \\
\hline & & & 1 & 3766 & CA \\
\hline & & & 1 & 2682 & $\mathrm{HI}$ \\
\hline & & & 1 & 1566 & SP \\
\hline & & & 1 & 1537 & LEV \\
\hline & & & 1 & 1289 & RD \\
\hline & & & 1 & 63 & ow \\
\hline & & & 1 & 60 & $\mathrm{CSH}$ \\
\hline & & & 1 & 38 & EmD \\
\hline & & & 1 & 24 & EoD \\
\hline & & & 1 & 20 & EoS \\
\hline & & & 1 & 17 & FStH \\
\hline & & & 1 & 8 & CStS \\
\hline & & & 1 & 7 & EmST \\
\hline & & & 1 & 2 & PS \\
\hline & & & 1 & 2 & $\mathrm{EcD}$ \\
\hline & & & 1 & 1 & EcS \\
\hline & & & 1 & 1 & $E$ \\
\hline & & & 1 & 1 & EGD \\
\hline
\end{tabular}



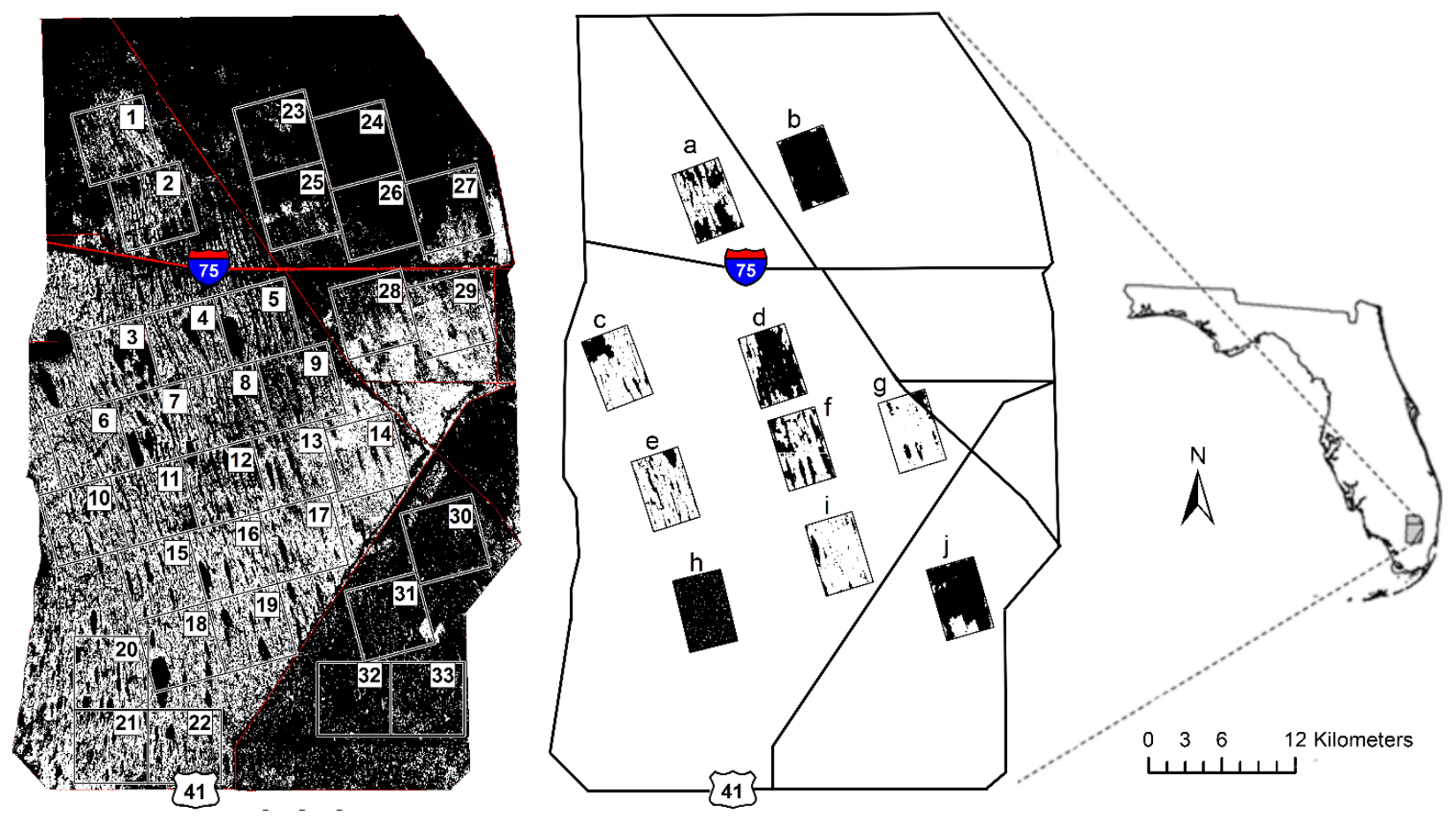

Figure S1 

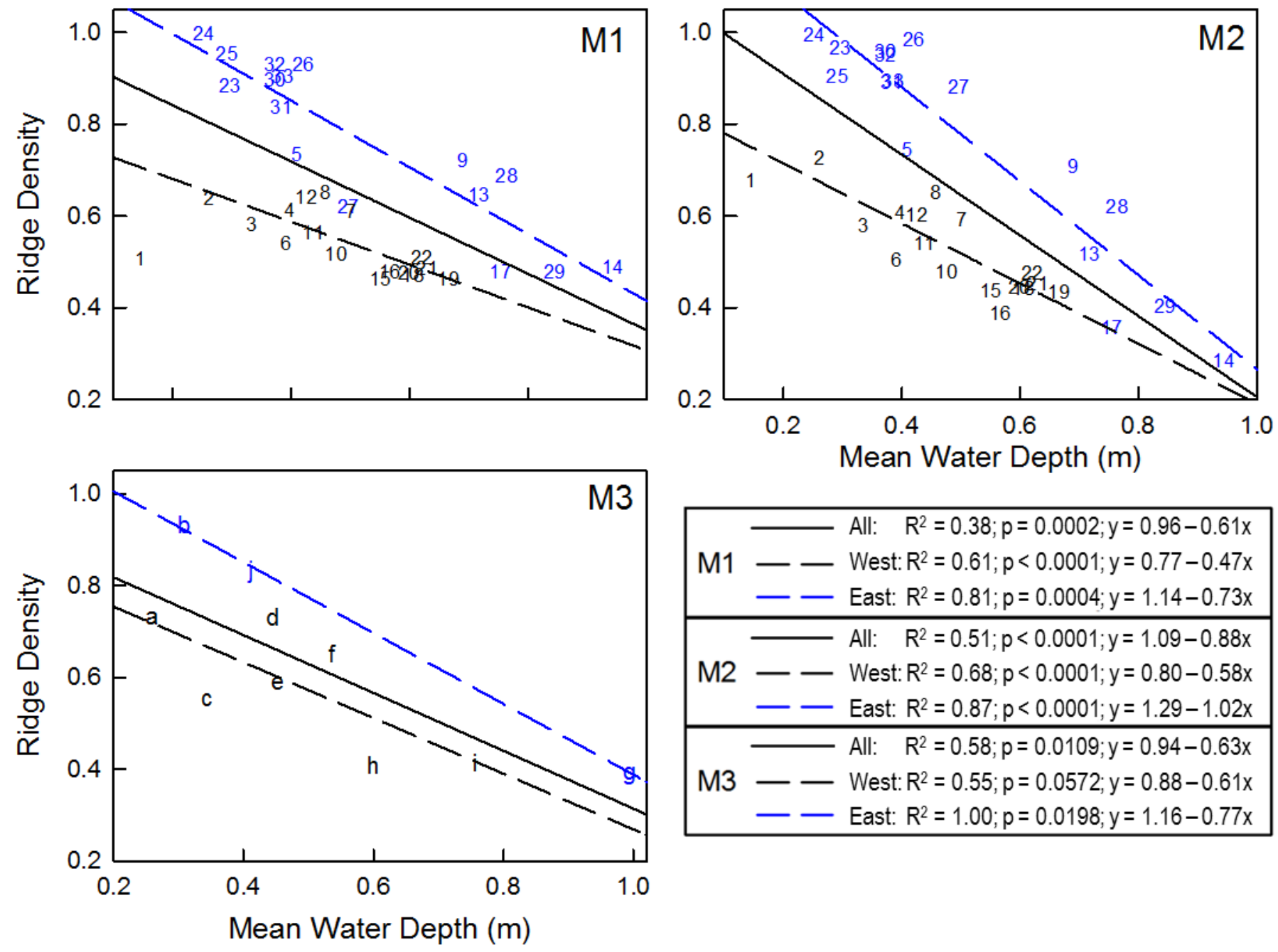

M1 - - All: $R^{2}=0.38 ; p=0.0002 ; y=0.96-0.61 x$
- West: $R^{2}=0.61 ; p<0.0001 ; y=0.77-0.47 x$
- East: $R^{2}=0.81 ; p=0.0004 ; y=1.14-0.73 x$
M2 - All: $R^{2}=0.51 ; p<0.0001 ; y=1.09-0.88 x$
- West: $R^{2}=0.68 ; p<0.0001 ; y=0.80-0.58 x$
M3 - East: $R^{2}=0.87 ; p<0.0001 ; y=1.29-1.02 x$
- All: $R^{2}=0.58 ; p=0.0109 ; y=0.94-0.63 x$
- West: $R^{2}=0.55 ; p=0.0572 ; y=0.88-0.61 x$
- East: $R^{2}=1.00 ; p=0.0198 ; y=1.16-0.77 x$

Figure S2 

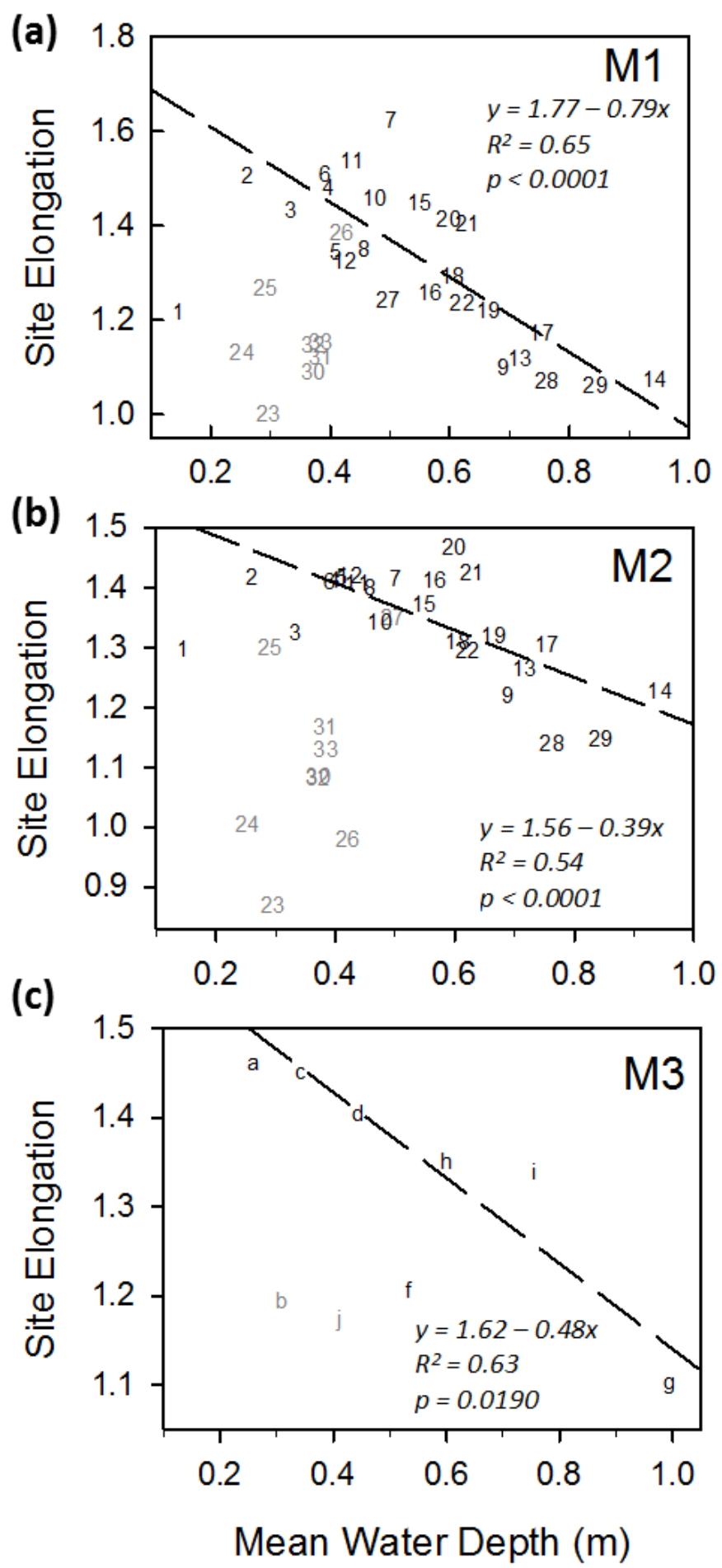

Figure S3 
(a)

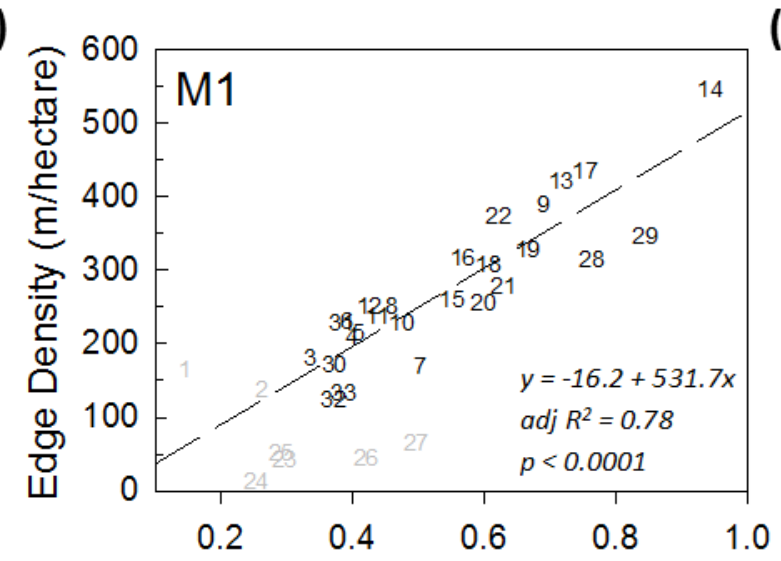

(d)

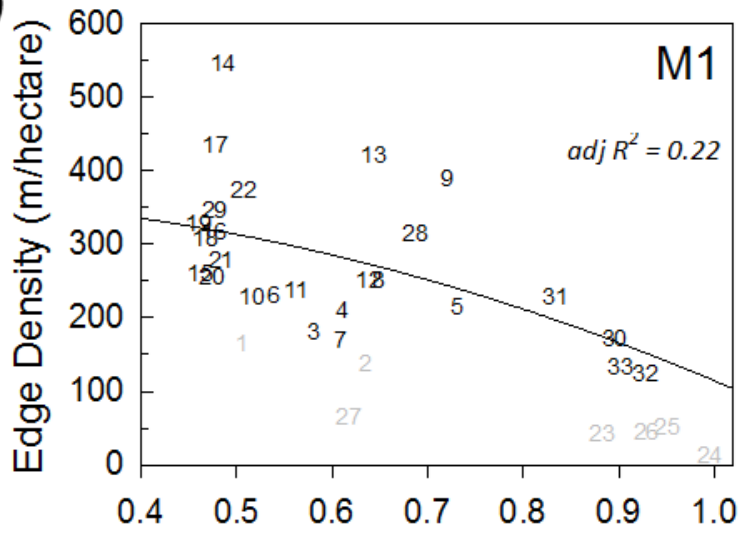

(e)

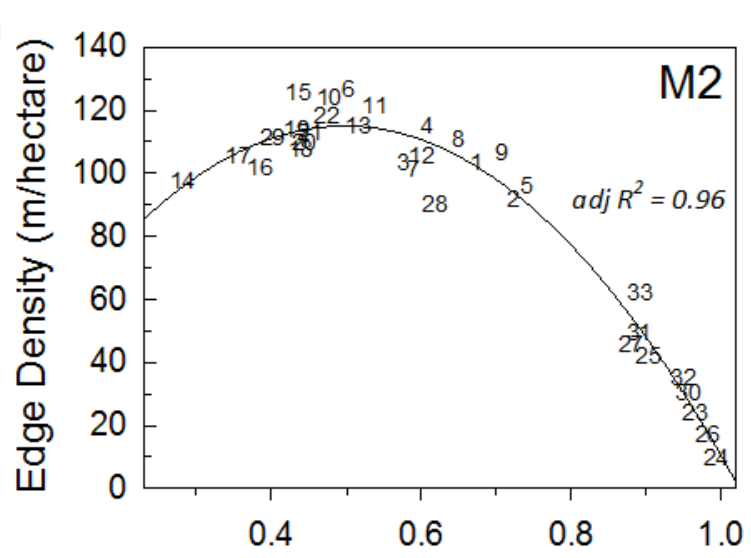

(f)

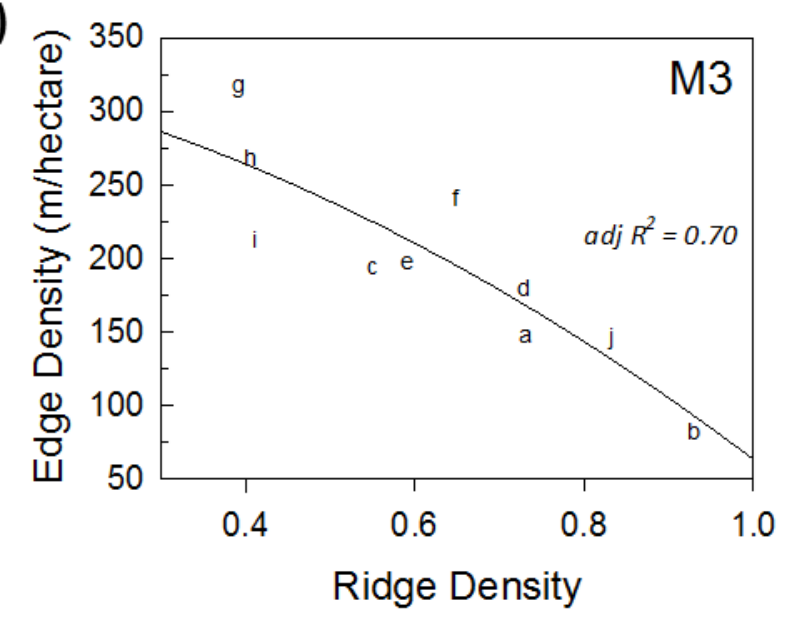

Fiure S4 
(a)

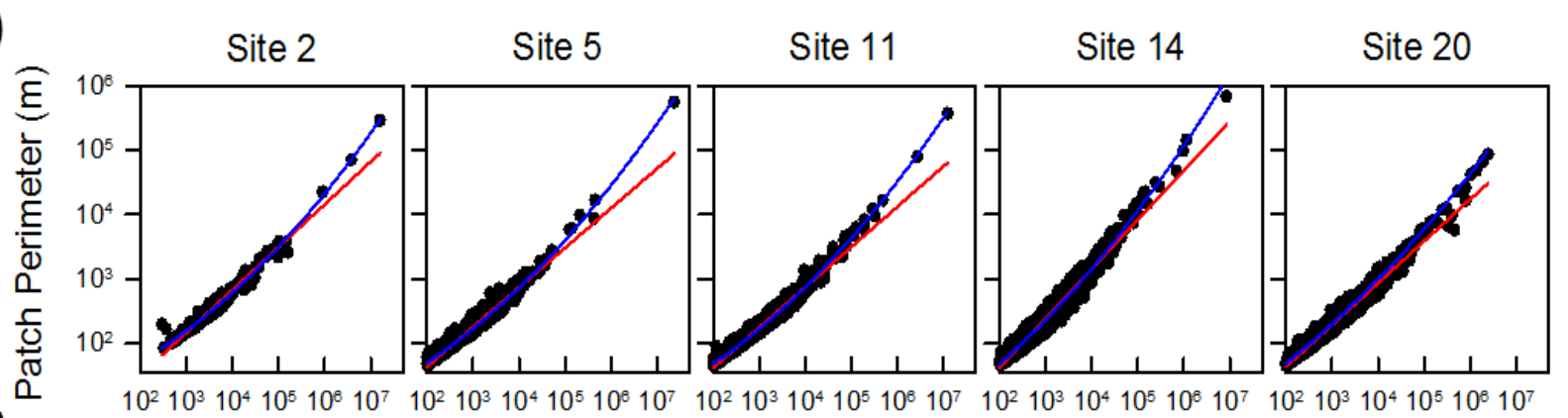

(b)

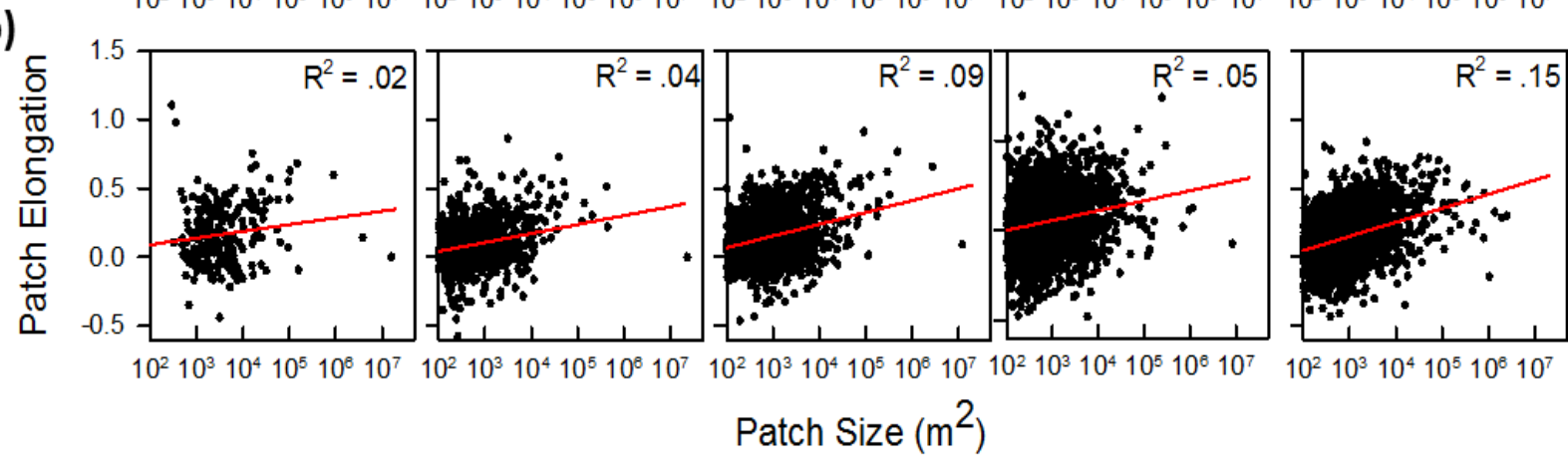

Figure S5 


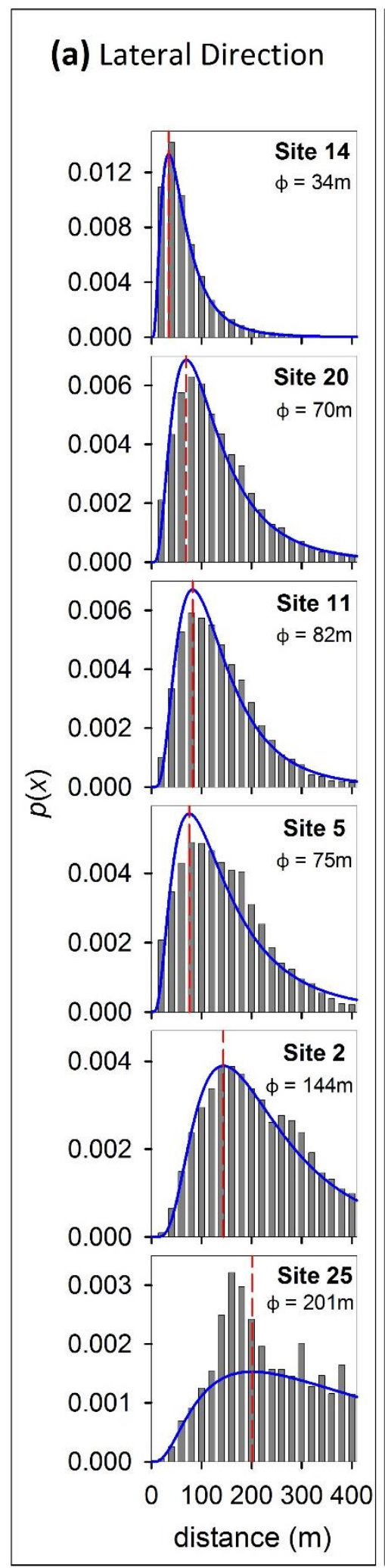

(b) Longitudinal Direction
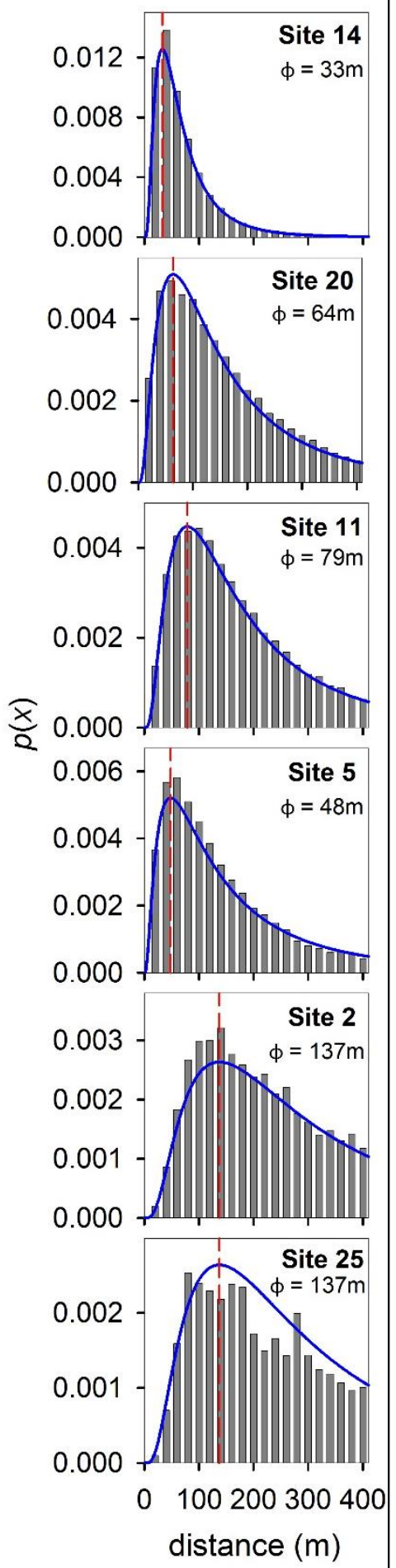

(c) Resolution Effects Example
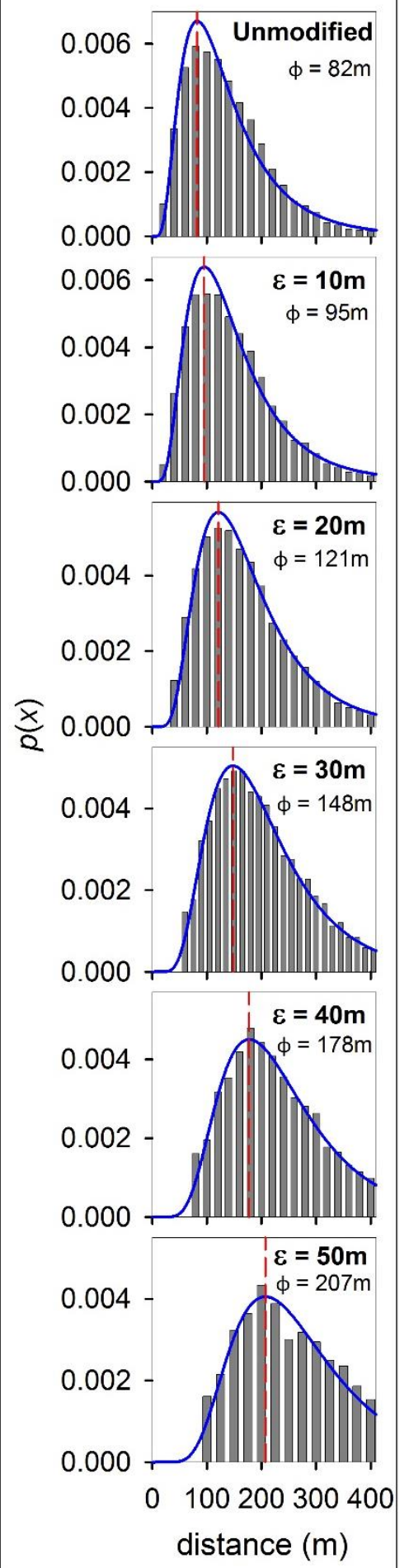

Figure S6 
a) lateral $r$ spectra

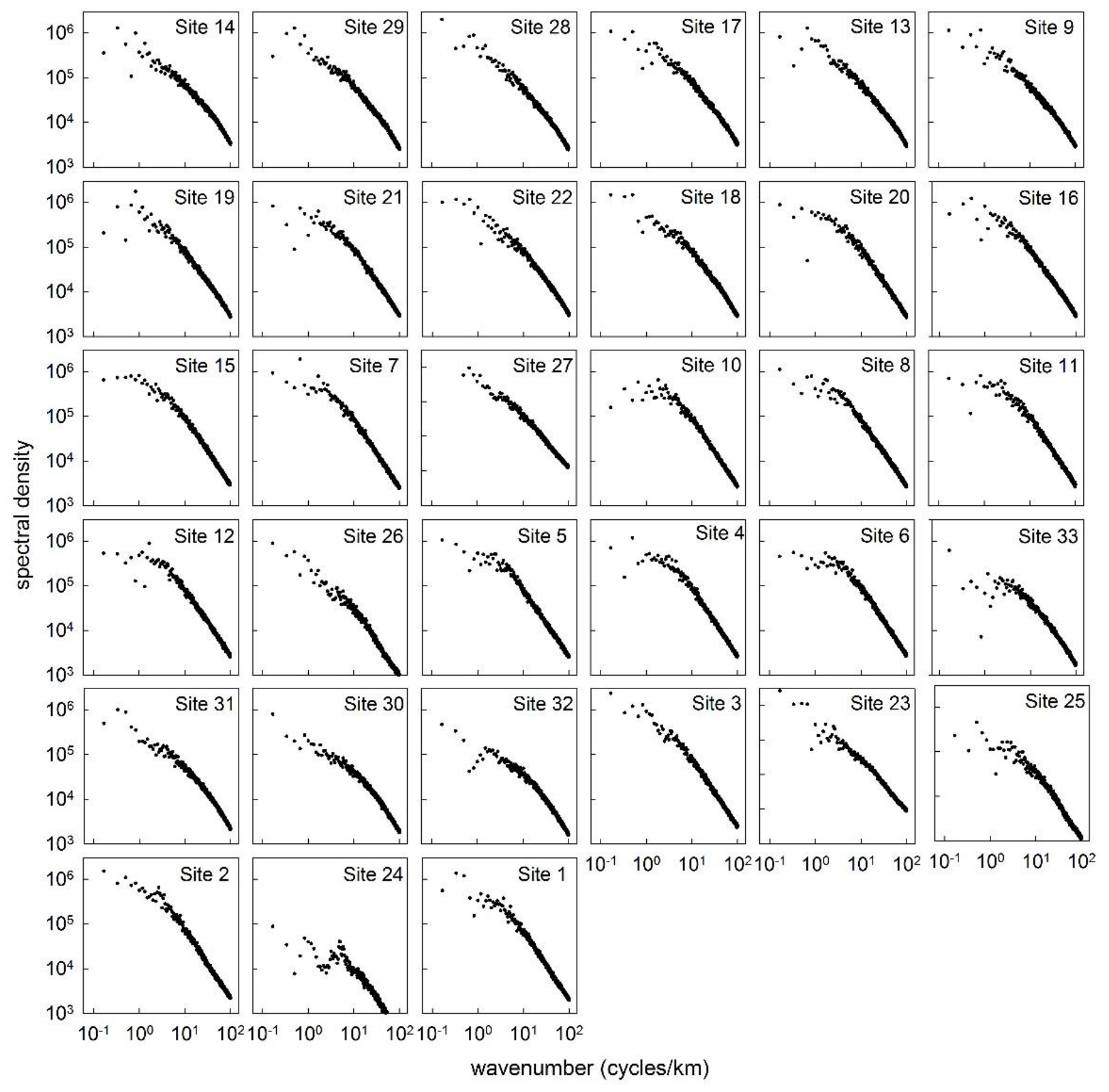

Figure S7A 
a) longitudinal $r$ spectra

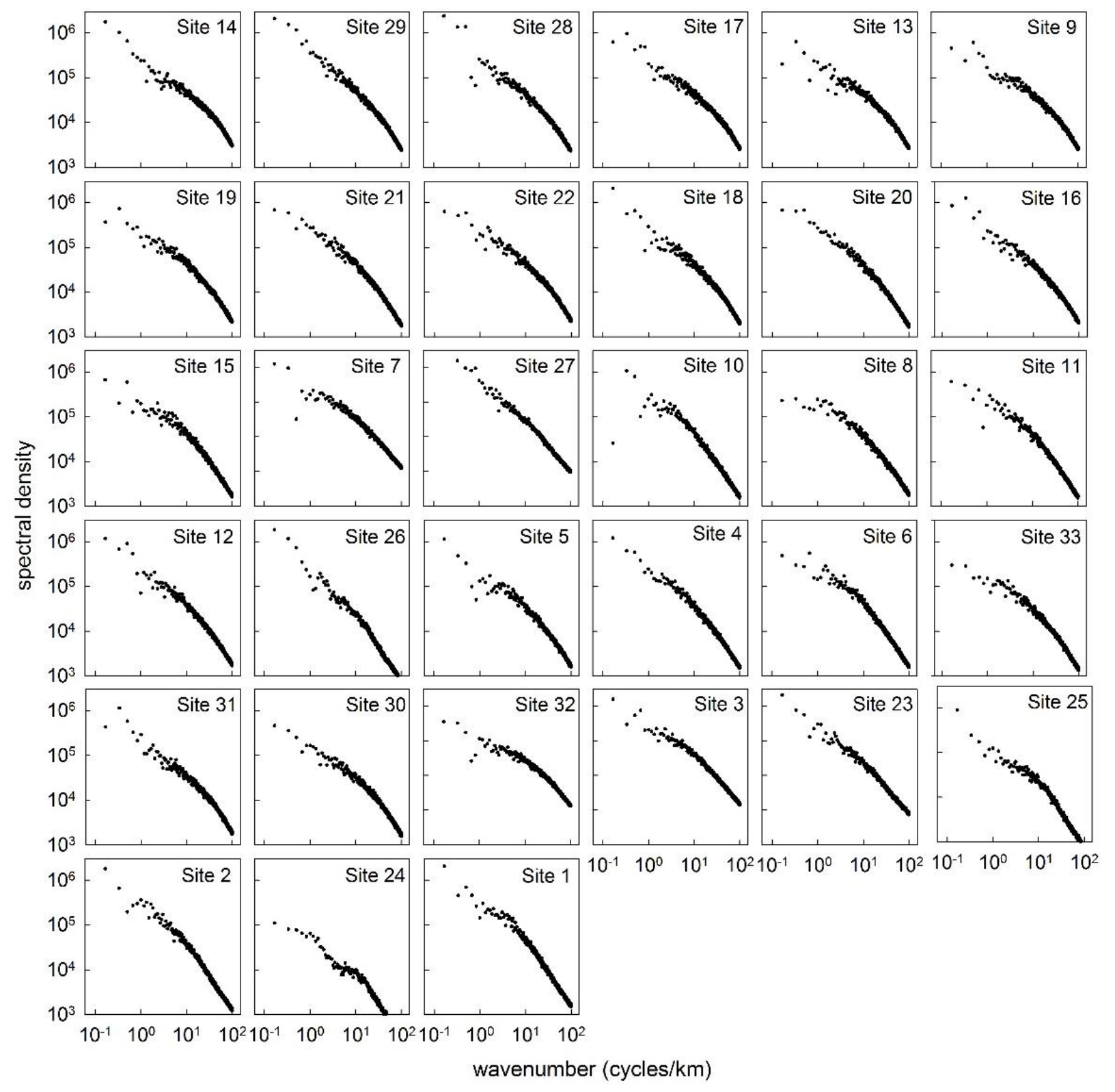

Figure S7B 